\title{
Influence of obesity on experimental periodontitis in rats: histopathological, histometric and immunohistochemical study
}

\author{
Elizangela Partata Zuza ${ }^{1,2}$ - Valdir Gouveia Garcia ${ }^{3}$ - Letícia Helena Theodoro ${ }^{3}$. \\ Edilson Ervolino $^{4}$ - Luiz Fernando Veloso Favero ${ }^{5}$ - Mariéllen Longo ${ }^{3}$. \\ Fernando Salimon Ribeiro ${ }^{5}$ • Alex Tadeu Martins ${ }^{5} \cdot$ Luís Carlos Spolidorio $^{6}$. \\ José Antônio Sampaio Zuanon ${ }^{6}$ • Benedicto Egbert Corrêa de Toledo ${ }^{7}$. \\ Juliana Rico Pires ${ }^{5}$
}

Received: 12 July 2016 / Accepted: 11 September 2017 /Published online: 19 September 2017

(C) Springer-Verlag GmbH Germany 2017

\begin{abstract}
Objectives This study assessed the influence of obesity on the progression of ligature-induced periodontitis in rats.

Materials and methods Forty-eight adult Wistar rats were randomly divided into two groups: the HL group $(n=24)$ was fed high-fat animal food to induce obesity, and the NL group $(n=24)$ was fed normolipidic animal food. Obesity was induced within a period of 120 days, and the induction of experimental periodontitis (EP) was subsequently performed for 30 days. The animals were euthanized after 7,15 , and 30 days, and the jaws were removed for histopathological, histometric,
\end{abstract}

Electronic supplementary material The online version of this article (https://doi.org/10.1007/s00784-017-2207-y) contains supplementary material, which is available to authorized users.

Elizangela Partata Zuza

elizangelazuza@yahoo.com.br

1 Department of Periodontology, School of Dentistry, Fluminense Federal University (UFF), Nova Friburgo, Rio de Janeiro, Brazil

2 Elizangela Partata Zuza, Instituto de Saúde de Nova Friburgo (ISNF) -UFF, Rua Dr. Silvio Henrique Braune, 22. Cep: 28625-650. Nova Friburgo, Rio de Janeiro, Brazil

3 Department of Surgery and Integrated Clinic, School of Dentistry, São Paulo State University (Unesp), Araçatuba, São Paulo, Brazil

4 Department of Basic Science, School of Dentistry, São Paulo State University (Unesp), Araçatuba, São Paulo, Brazil

5 Department of Dentistry, School of Dentistry, Educational Foundation of Barretos (Unifeb), Barretos, São Paulo, Brazil

6 Department of Physiology and Pathology, School of Dentistry, São Paulo State University (Unesp), Araraquara, São Paulo, Brazil

7 Department of Diagnosis and Surgery, School of Dentistry, São Paulo State University (Unesp), Araraquara, Brazil and immunohistochemical analyses. Tartrate-resistant acid phosphatase (TRAP), receptor activator of nuclear factor kappa beta ligand (RANKL), and osteoprotegerin (OPG) were analyzed via immunolabeling.

Results Histological findings indicated that the inflammation was more extensive and lasted longer in the HLEP; however, advanced destruction also occurred in the NL/EP. Greater bone loss was verified in the HL/EP group $(2.28 \pm 0.35)$ in the period of 7 days than in the NL/EP group $(1.2 \pm 0.29)$. High immunolabeling was identified in the HL/EP group in the initial periods for RANKL and TRAP, whereas the NLEP group presented with moderate immunolabeling for both factors. The HL/EP and NL/EP groups showed low immunolabeling for OPG.

Conclusions Obesity induced by a high-fat diet influenced alveolar bone metabolism when associated with experimental periodontitis and caused a more severe local inflammatory response and alveolar bone loss.

Clinical relevance Obesity is related to greater alveolar bone loss and an accentuated local inflammatory response, which may be reflected in the clinical severity of periodontitis and dental loss.

Keywords Periodontitis · Alveolar bone loss · Obesity $\cdot$ Body weight $\cdot$ Rats $\cdot$ Inflammation

\section{Introduction}

Periodontitis is a chronic infectious and inflammatory disease caused by periodontopathogens, which lead to the destruction of the tissues that support the teeth [1]. Serum levels of pro-inflammatory cytokines, such as interleukin-1beta, interleukin-6, and tumor necrosis factor- 
alpha (TNF), have been shown to be high in patients with severe chronic periodontitis [2]. Moreover, these cytokines are elevated in the adipose tissues of obese individuals [3].

Adipose cells secrete bioactive molecules collectively referred to as adipokines (leptin and adiponectin), classical cytokines (tumor necrosis factor-alpha and interleukin-6), proteins involved in vascular hemostasis (inhibitor of plasminogen activator-1), regulators of blood pressure (angioten sinogen), promoters of angiogenesis (vascular endothelial growth factor), and the respondents of acute phase protein (C-reactive protein) [4].

Based on evidence that adipose tissue serves as a reservoir of inflammatory cytokines, it is possible to suggest that an increase in body fat results in a larger and more active inflammatory response of the host in cases of periodontal disease $[4,5]$. The potential causal relationship between obesity and periodontitis, as well as the potential biological mechanisms, must be established. However, findings have pointed to the involvement of similar pathways in the pathophysiology of obesity, periodontal disease, and other related inflammatory diseases [6].

Systematic literature reviews and meta-analyses of clinical trials have supported the idea that obesity may be considered a risk factor for periodontitis [7, 8]. Findings from animal models indicated that obesity potentially influences the pathogenesis of experimental periodontitis and led to greater alveolar bone loss [9, 10], whereas other findings showed no influence of obesity on bone loss in rats with ligature-induced periodontitis [11-13]. The present study aims to assess the influence of induced obesity on the bone loss, local inflammatory response, osteoclast recruitment and main regulators of osteoclastogenesis, and osteoclastic activation in rats with ligature-induced periodontitis.

\section{Materials and methods}

\section{Selection of animals}

Seventy-one adult male rats (Rattus norvegicus albinus, Wistar) were maintained for 2 weeks in a vivarium at the University Center of Educational Foundation of Barretos (UNIFEB), and a routine with an airconditioned environment and a 12-h light-dark cycle was provided. The study protocol was approved by the Committee for Animal Experimentation of Araçatuba (CEUA, n. 00263-2012).

The animals were randomly divided into two groups of diet: the NL group - animals fed standard normolipidic animal food (Presence rats and mice®, São Lourenço da Mata, Pernambuco, Brazil) and the HL group - animals fed a high- fat diet (PragSoluções, Jaú, Sao Paulo, Brazil). Initially, 41 rats were allocated to the HL group and 30 rats were allocated to the NL group during the period from October to December 2012. A larger number of rats were allocated to the HL group for the selection of animals prone to obesity 60 days after high-fat diet initiation, according to the study of Cabeço et al. [14].

Of the 41 animals in the HL group, 12 animals were excluded from the study because they had resistance to obesity as detected by low weight gain (1.75-6.75\%) [14], 3 animals were excluded because of blood glucose levels $>300 \mathrm{mg} / \mathrm{dl}$ $[15,16]$, and 2 animals died. Of the 30 animals in the NL group, 2 animals were excluded because of glucose levels $>$ $300 \mathrm{mg} / \mathrm{dl}$, and 4 rats died. Thus, 24 animals in the HL group and 24 rats in the NL group were included in the study at day 0 , which was considered baseline. The experimental design is shown in Fig. 1.

\section{Obesity induction protocol}

The animals in the HL group were fed a high-fat diet in which the composition of macronutrients was $35 \%$ ethereal extract (fat), 26\% raw protein, 25\% carbohydrates, $8 \%$ mineral material, and $6 \%$ raw fiber. The animals were fed the high-fat diet for a period of 120 days (16 weeks) to induce obesity, whereas the NL group was fed throughout the same period with normolipidic animal food that contained $42.8 \%$ carbohydrate, $23 \%$ raw protein, $4 \%$ ethereal extract, $10 \%$ mineral material, and $5 \%$ raw fiber. The animals were weighed weekly throughout the experimental period, and the normolipidic or high-fat animal food was provided until the end of the experiment. The animals were considered obese if they had a weight difference of at least $15 \%$ relative to the weight of the control group animals up to the period of 120 days [11]. The Lee index was also used as a predictor of obesity in rats and was calculated by the ratio of the cube root of body weight $(\mathrm{g}) /$ nasoanal length $(\mathrm{cm}) \times 1000$.

\section{Protocol for induction of experimental periodontitis}

Anesthesia via intramuscular injection with ketamine hydrochloride $\left(70 \mathrm{mg} / \mathrm{kg}\right.$; Vetaset ${ }^{\circledR}$; Fort Dodge Animal Health, Fort Dodge, IA, USA) and xylazine hydrochloride (6 mg/kg; Coopazine, Coopers, Sao Paulo, SP, Brazil) was performed for the induction of experimental periodontitis (EP). The animals were positioned on the operating table to facilitate access to the molars. With the aid of a probe and clamp modified for this purpose, a number 24 cotton thread (Coats Corrente, Sao Paulo, SP, Brazil) (Cotton chain number 10) was placed around the right lower first molars and maintained with a knot for the induction of EP $[17,18]$. The molar on the left side of each animal served as a control 
Fig. 1 Experimental design

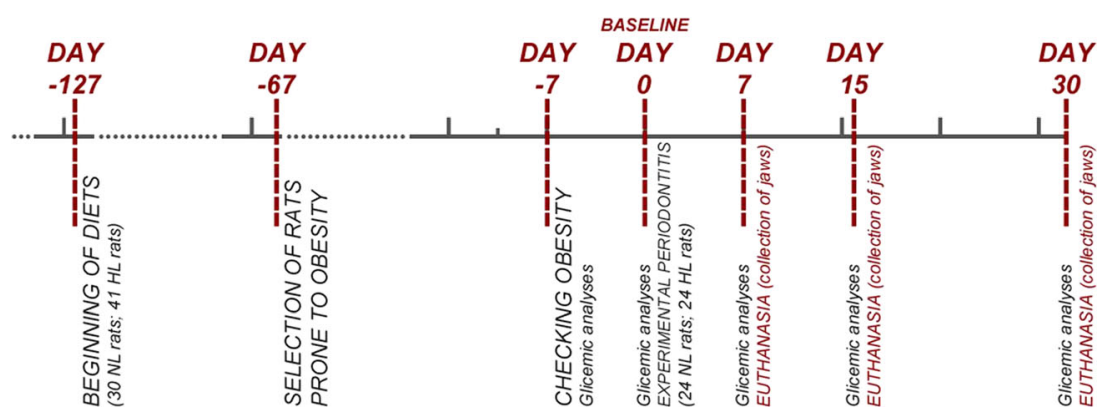

(C) to assess the systemic effects of obesity. Seven days after the end of the obesity induction, the day of the ligature installation was considered the baseline (Fig. 2).

\section{Experimental periods}

The animals were euthanized at 7,15 , or 30 days after the baseline. Eight animals from each group were euthanized by anesthetic overdose (Thiopental $150 \mathrm{mg} / \mathrm{kg}$; Cristalia, chemicals pharmaceuticals Ltd., Itapira, SP, Brazil). The jaw of each animal was removed, separated in half, and fixed in $4 \%$ buffered formaldehyde for $48 \mathrm{~h}$.

\section{Analysis of blood glucose levels}

Blood samples from the tail were collected in tubes that contained heparin (Heparin Sodium; Roche, Sao Paulo, SP, Brazil) [19]. Following centrifugation of the blood to obtain plasma, glucose was measured via enzymatic method of a glucose-oxidase RA-XT autoanalyzer (Technicon Bayer Diagnostics, Dublin, Ireland). The glycemia was measured $(\mathrm{mg} / \mathrm{dl})$ at day -7 , baseline (day 0 ), and each experimental time point of 7, 15, and 30 days, and the animals' weights were obtained at the same time

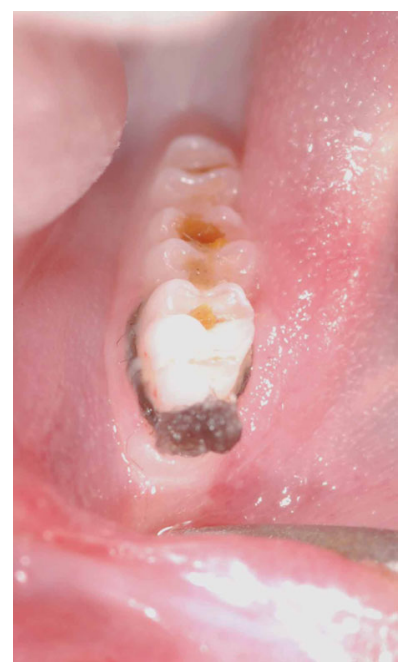

Fig. 2 Clinical aspect of the first lower molar 7 days after ligature installation points [19]. At day -7 , three animals that presented glucose levels $>300 \mathrm{mg} / \mathrm{dl}$ were excluded from the HL group, and two rats were excluded from the NL group. At baseline until the end of the experiment, no animal had a glucose level greater than $300 \mathrm{mg} / \mathrm{dl}$.

\section{Laboratory processing}

After the fixation period, the specimens were demineralized in $10 \%$ EDTA (Sigma Chemical Co., USA). Processing of the samples was conducted in a conventional manner, embedded in paraffin, and sectioned with $4 \mu \mathrm{m}$ thickness in a semi-serial form in a mesiodistal direction. Some specimens were stained using the hematoxylin and eosin (HE) method, and other specimens were submitted to the indirect immunoperoxidase method [17]. For the immunohistochemical reactions, histological sections were deparaffinized in xylene and rehydrated in decreasing series of ethanol. The antigen retrieval was performed by immersion of the histological sections in a buffer solution (Diva Decloaker®; Biocare Medical, Concord, CA, USA) in a pressurized chamber (Decloaking Chamber®; Biocare Medical) at $95{ }^{\circ} \mathrm{C}$ for $10 \mathrm{~min}$. The sections were washed with $0.1 \mathrm{M}$ phosphate buffer saline ( $\mathrm{pH}$ 7.4) at the end of each stage of the immunohistochemical reaction. The histological sections were immersed in $3 \%$ hydrogen peroxide for $1 \mathrm{~h}$ and $1 \%$ bovine serum albumin for $12 \mathrm{~h}$ to block the endogenous peroxidase activity and the non-specific sites, respectively. The histological sections that contained samples of the groups were divided into three baths, and each bath was incubated with one of the following primary antibodies: goat anti-TRAP (1:200, SC 30833, Santa Cruz Biotechnology, Santa Cruz, CA, USA), goat anti-RANKL (1:100, SC 7628, Santa Cruz Biotechnology, Santa Cruz, CA, USA), or goat anti-OPG (1:100, SC 8468, Santa Cruz Biotechnology, Santa Cruz, CA, USA). The primary antibodies were diluted in phosphate buffered saline with $0.1 \%$ Triton X-100 (Sigma Chemical Co., USA) and were placed in a humid chamber for $24 \mathrm{~h}$. The histological sections were incubated with a biotinylated secondary antibody for $2 \mathrm{~h}$ and were subsequently treated with horseradish peroxidase-conjugated and streptavidin for 1 h (Universal Dako Labeled Streptavidin-Biotin kit@; Dako Laboratories, Carpinteria, CA, USA). The reactions were 
Table 1 Parameters assessed in histologic analysis of the mandibular first molar according to groups and time point and distribution of specimens per scores

Parameters and respective scores

Percentage of the animals

Experimental groups

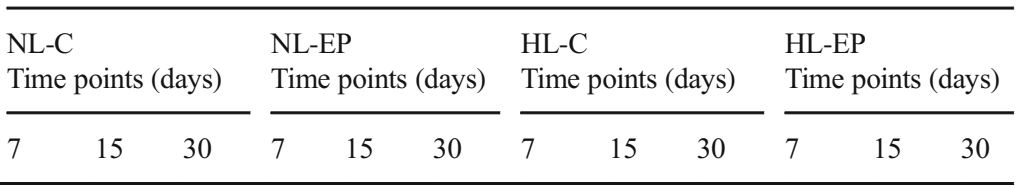

Intensity of local inflammatory infiltrate

(0) Absence of inflammation

(1) Small amount of inflammatory cells

(2) Moderate amount of inflammatory cells

$\begin{array}{llllllllllll}0 \% & 0 \% & 0 \% & 0 \% & 0 \% & 0 \% & 0 \% & 0 \% & 0 \% & 0 \% & 0 \% & 0 \%\end{array}$

(3) Large amount of inflammatory cells

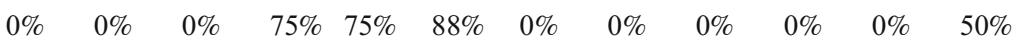

$\begin{array}{llllllllllll}0 \% & 0 \% & 0 \% & 25 \% & 25 \% & 12 \% & 0 \% & 0 \% & 0 \% & 100 \% & 100 \% & 50 \%\end{array}$

Extension of inflammatory infiltrate

(0) Absence of inflammation

$\begin{array}{llllllllllll}100 \% & 100 \% & 100 \% & 0 \% & 0 \% & 0 \% & 100 \% & 100 \% & 100 \% & 0 \% & 0 \% & 0 \%\end{array}$

(1) Extending to part of the connective tissue of the furcation area

(2) Extending to the whole connective tissue of the furcation area

(3) Extending to the whole connective tissue and to the bone tissue of the furcation area

External root resorption (Cementum and dentin)
(0) Absent
(1) Only inactive resorption areas
(2) Few active resorption areas
(3) Many active resorption areas

$\begin{array}{llllllllllll}0 \% & 0 \% & 0 \% & 0 \% & 0 \% & 0 \% & 0 \% & 0 \% & 0 \% & 0 \% & 0 \% & 0 \% \\ 0 \% & 0 \% & 0 \% & 75 \% & 75 \% & 100 \% & 0 \% & 0 \% & 0 \% & 0 \% & 0 \% & 12 \% \\ 0 \% & 0 \% & 0 \% & 25 \% & 25 \% & 0 \% & 0 \% & 0 \% & 0 \% & 100 \% & 100 \% & 88 \%\end{array}$

Alveolar bone resorption

(0) Within normality patterns

(1) Small amount of resorption bone

(2) Moderate amount of resorption bone

(3) Large amount of resorption bone

$\begin{array}{llllllllllll}100 \% & 100 \% & 100 \% & 0 \% & 0 \% & 0 \% & 100 \% & 100 \% & 100 \% & 0 \% & 0 \% & 0 \% \\ 0 \% & 0 \% & 0 \% & 0 \% & 0 \% & 0 \% & 0 \% & 0 \% & 0 \% & 0 \% & 0 \% & 0 \% \\ 0 \% & 0 \% & 0 \% & 75 \% & 100 \% & 100 \% & 0 \% & 0 \% & 0 \% & 0 \% & 50 \% & 50 \% \\ 0 \% & 0 \% & 0 \% & 25 \% & 0 \% & 0 \% & 0 \% & 0 \% & 0 \% & 100 & 50 \% & 50 \%\end{array}$

Pattern of structuration of the connective tissue

(0) Moderate amount of fibroblasts and large amount of collagen fibers (dense connective tissue)

(1) Moderate amount of both fibroblasts and collagen fibers

(2) Small amount of both fibroblasts and collagen fibers; presence of intersticial edema

(3) Severe tissue breakdown with intersticial edema and necrotic areas

$\begin{array}{llllllllllll}100 \% & 100 \% & 100 \% & 0 \% & 0 \% & 0 \% & 100 \% & 100 \% & 100 \% & 0 \% & 0 \% & 0 \% \\ 0 \% & 0 \% & 0 \% & 0 \% & 0 \% & 0 \% & 0 \% & 0 \% & 0 \% & 0 \% & 0 \% & 0 \% \\ 0 \% & 0 \% & 0 \% & 50 \% & 75 \% & 100 \% & 0 \% & 0 \% & 0 \% & 0 \% & 0 \% & 50 \% \\ 0 \% & 0 \% & 0 \% & 50 \% & 25 \% & 0 \% & 0 \% & 0 \% & 0 \% & 100 \% & 100 \% & 50 \%\end{array}$

Pattern of structuration of the bone alveolar

(0) Bone trabeculae with regular contour coated with active osteoblasts, including areas of new bone formation

(1) Bone trabeculae with irregular contour coated with active osteoblasts and osteoclasts

(2) Bone trabeculae with irregular contour coated with active osteoclasts

(3) Areas of necrotic bone and bone trabeculae with irregular contour coated with active osteoclasts

$\begin{array}{llllllllllll}100 \% & 100 \% & 100 \% & 0 \% & 0 \% & 0 \% & 100 \% & 100 \% & 100 \% & 0 \% & 0 \% & 0 \% \\ 0 \% & 0 \% & 0 \% & 0 \% & 0 \% & 0 \% & 0 \% & 0 \% & 0 \% & 0 \% & 0 \% & 0 \% \\ 0 \% & 0 \% & 0 \% & 50 \% & 75 \% & 75 \% & 0 \% & 0 \% & 0 \% & 0 \% & 0 \% & 12 \% \\ 0 \% & 0 \% & 0 \% & 50 \% & 25 \% & 25 \% & 0 \% & 0 \% & 0 \% & 100 \% & 100 \% & 88 \%\end{array}$

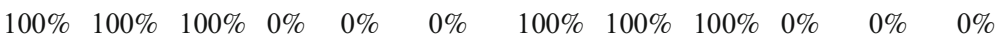
$\begin{array}{llllllllllll}0 \% & 0 \% & 0 \% & 0 \% & 0 \% & 0 \% & 0 \% & 0 \% & 0 \% & 0 \% & 0 \% & 0 \%\end{array}$

$\begin{array}{llllllllllll}0 \% & 0 \% & 0 \% & 50 \% & 75 \% & 75 \% & 0 \% & 0 \% & 0 \% & 0 \% & 0 \% & 12 \%\end{array}$

$\begin{array}{llllllllllll}0 \% & 0 \% & 0 \% & 50 \% & 25 \% & 25 \% & 0 \% & 0 \% & 0 \% & 100 \% & 100 \% & 88 \%\end{array}$ 
developed using a 3,3'diaminobenzidine tetra-hydrochloride (DAB) chromogen (DAB chromogen kit ${ }^{\circledR}$, Dako Laboratories, USA) and counterstained with Harris hematoxylin. The negative controls were treated with the previously described procedures without the primary antibodies [17].

\section{Histologic analysis}

The histologic analysis was conducted with the use of HE staining, and it was performed by a certified histologist (EE) using a light microscope. The parameters analyzed were as follows: intensity of local inflammatory response, extension of inflammatory infiltrate and external root resorption (cementum and dentin), alveolar bone loss, pattern of structuration of the connective tissue, and pattern of structuration of the alveolar bone [20].

\section{Histometric analysis}

For the analysis of the inter-radicular bone level, we employed HE staining. The area expressed in $\mathrm{mm}^{2}$ of bone loss (BL) in the furcation region was measured using an image analysis system (Axiovision 4.8.2; Carl Zeiss MicroImaging GmbH, 07740 Jena, Germany). After exclusion of the first and last sections in which the furcation region was evident, five equidistant sections of each tooth were selected for histometric analysis and photographed with a digital camera attached to the microscope (AxioStar Plus; Carl Zeiss MicroImaging GmbH, 07740 Jena, Germany). BL was assessed by measuring the extent of the area between the bone crest and the surface of the furcation ceiling cementum. The selection of the histological sections for histological and histometric analyses were performed by a trained examiner who was blind to the experimental conditions (ML). The BL of each specimen was measured three times by the same examiner and on different days. The three measurements obtained were statistically analyzed for concordance analysis with a 5\% significance level (Kappa). The average values were compared statistically [16].

\section{Immunohistochemical analysis}

The histological sections were examined under bright-field microscopy. A calibrated examiner (LHT) who was blinded to the experiment selected the sections for immunohistochemical analysis. Immunohistochemical analysis was performed by one blinded examiner (EE). Quantitative analyses of the immunolabeled cells were performed for TRAP in an area of $1000 \times 1000 \mu \mathrm{m}$ in the center of the interradicular septum of the left first mandibular. The crown outline of this area was the alveolar bone crest, from which it extended apically for a distance of $1000 \mu \mathrm{m}$. The value of each section was measured three times by the same examiner on different days to reduce the variations in the data. RANKL and OPG were analyzed in the central area of the furcation region of an area of $3200 \times 300 \mu \mathrm{m}$ under $\times 400$. The area coronary limit was the furcation top, which was extended apically for the distance of $3200 \mu \mathrm{m}$. Semi-quantitative RANKL and OPG analyses were performed for the alveolar bone and periodontal ligament in this area. Three histological sections were used for each animal, and the criterion for considering the immunolabeling for RANKL and OPG were as follows [17, 21]: score 0 , absence of immunolabeling (total absence of immunoreactivity-IR - in the cells); score 1 , low immunolabeling (IR in approximately $1 / 4$ of the cells per area); score 2, moderate immunolabeling (IR in approximately $1 / 2$ of the cells per area); and score 3 , high immunolabeling (IR in approximately $3 / 4$ of the cells per area).

\section{Intra-examiner reproducibility}

Prior to performing the histometric and immunohistochemical analyses, the examiner was trained (ML and EE) through dual measurements of 24 specimens with a 1-week interval. A paired $t$ test was calculated, and no difference was identified between the mean values of the two measuring times $(p>0.05)$. In addition, Pearson's correlation coefficient showed a high correlation $(0.95)$ between the two measuring times for both the histometric analysis and the immunohistochemical analysis.

\section{Statistical analysis}

Power calculations showed that with a sample of 8 $(p<0.05)$, the power of the study was 95\%. The hypothesis that the BL and the number of TRAP-positive cells in the furcation region would not differ among the groups and periods was tested. The normality of the histometric data of the BL, TRAP-positive cells, and blood glucose values was analyzed using the Shapiro-Wilk test. The intra- and intergroup analyses were performed through the analysis of variance test (ANOVA) $(P<0.05)$. When the ANOVA detected a statistically significant difference, multiple comparisons were made using Tukey's test $(P<0.05)$. The percentage of fattening was calculated, and a statistically significant difference was identified by the Binomial test. Differences in the weight and Lee Index were analyzed via $t$ tests $(P<0.05)$. The non-parametric Kruskal-Wallis test was used to compare the ranks (RANKL and OPG) of the groups. This test was followed by the non-parametric Dunn test when the Kruskal-Wallis test suggested a significant difference among the groups $(p<0.05)$. Statistical analyses were conducted using Bioestat software (Bioestat 5.0, Manaus, AM, Brazil). 


\section{Results}

\section{Fattening and glycemic analyses}

The results indicated higher levels of body weight and the Lee Index in the HL animals than the NL animals $(p<0.01$; Supplementary material). The mean $( \pm \mathrm{SD})$ blood glucose levels $(\mathrm{mg} / \mathrm{dl})$ were analyzed at baseline $(\mathrm{NL}=144.9 \pm 57.5 ; \mathrm{HL}=118.1 \pm 22.3), 7$ days $(\mathrm{NL}=147.7 \pm 62.4 ; \mathrm{HL}=120.4 \pm 27), 15$ days $(\mathrm{NL}=263.8 \pm 29 ; \mathrm{HL}=240.3 \pm 40.5)$, and 30 days $(\mathrm{NL}=174.2 \pm 6.6 ; \mathrm{HL}=196.85 \pm 61.9)$. There were no statistically significant differences between the NL and HL groups in all experimental time points ( $p \geq 0.05$ ).

\section{Histological analysis}

Table 1 shows the distribution of specimens according to each parameter assessed in the histologic assessment. In the control (C) groups that received the normolipidic $\operatorname{diet}(\mathrm{NL} / \mathrm{C})$ and high-fat diet (HL/C), the histological characteristics of the periodontal tissue exhibited a normal pattern and showed a similarity in all experimental periods. The bone trabeculae

Fig. 3 Photomicrographs highlighting the periodontal tissues of rats treated with a normolipidic diet (group NL/C; a-c) or high-fat diet (group HL/C; d-f). Histological characteristics were similar in both groups, showing aspects of normality. Abbreviations and symbols: ab, alveolar bone; ce, radicular cementum; pdl, periodontal ligament. Staining: hematoxylin and eosin (HE). Original magnification $\times 160$. Scale bars $120 \mu \mathrm{m}$
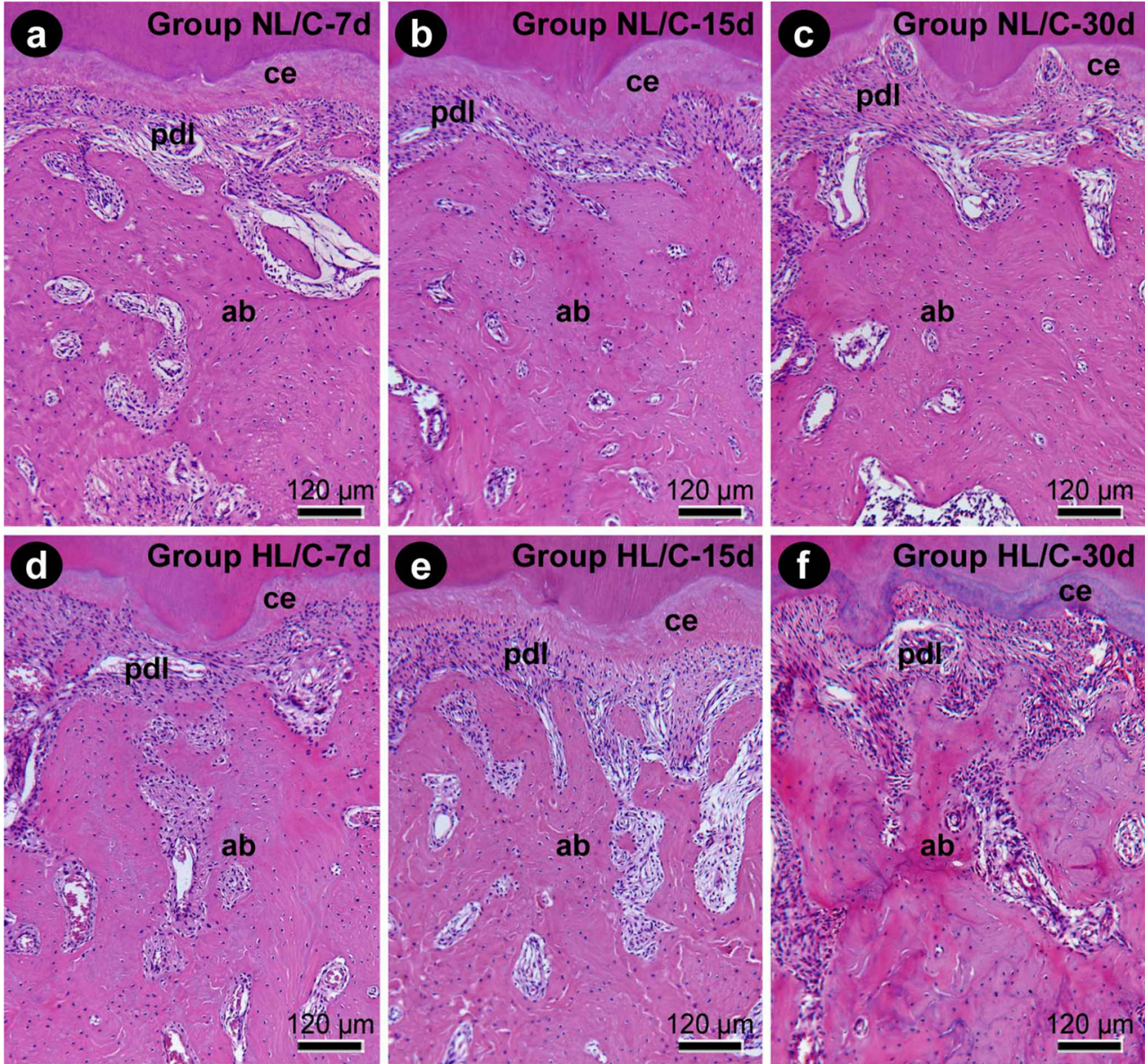

were fairly thick and were delimiting small medullary spaces filled by bone hematogenous marrow (Fig. 3).

The histological characteristics presented by the NL/EP (Fig.4) and HL/EP (Fig.5) groups were similar; however, the degree of tissue disruption and the magnitude of the local inflammatory response were more marked, particularly in the early stages in the HL/EP group. On the seventh day, an intense inflammatory infiltration was identified throughout the connective tissue of the furcation area. All specimens in this group had spikes of necrotic bone, with large dimensions in the HL/EP group, which were surrounded by inflammatory cells in the furcation area. The interradicular septum was substantially reduced, very rough, and had a very thin height of bone trabeculae; on the surface, a substantial number of active osteoclasts were identified, even in the later periods. On the 15th and 30th days, the inflammation remained intense; however, compared with the 7 th day, in both groups, there was a reduction in the volume occupied by inflammatory infiltration.

\section{Histometric analysis}

Table 2 shows higher levels of BL for both groups with the normolipidic diet (NL/EP) and the high-fat diet (HL/EP) with 
Fig. 4 Histopathological appearance in the furcation region of the mandibular molar focusing on the evolution of EP in rats with a normolipidic diet (group NL/ EP). Histopathological features after 7 (a and $\mathbf{d}), 15$ (b and $\mathbf{e}$ ), and 30 (c and $\mathbf{f}$ ) days post-ligature placement. Abbreviations and symbols: ab, alveolar bone; $\mathrm{nb}$, necrotic bone; asterisks: inflammatory infiltrate. Staining: hematoxylin and eosin (HE). Original magnification: a-c, $\times$ $100 ; \mathbf{d}-\mathbf{f}, \times 250$. Scale bars: $\mathbf{a}-\mathbf{c}$, $250 \mu \mathrm{m} ; \mathbf{d}-\mathbf{f}, 100 \mu \mathrm{m}$
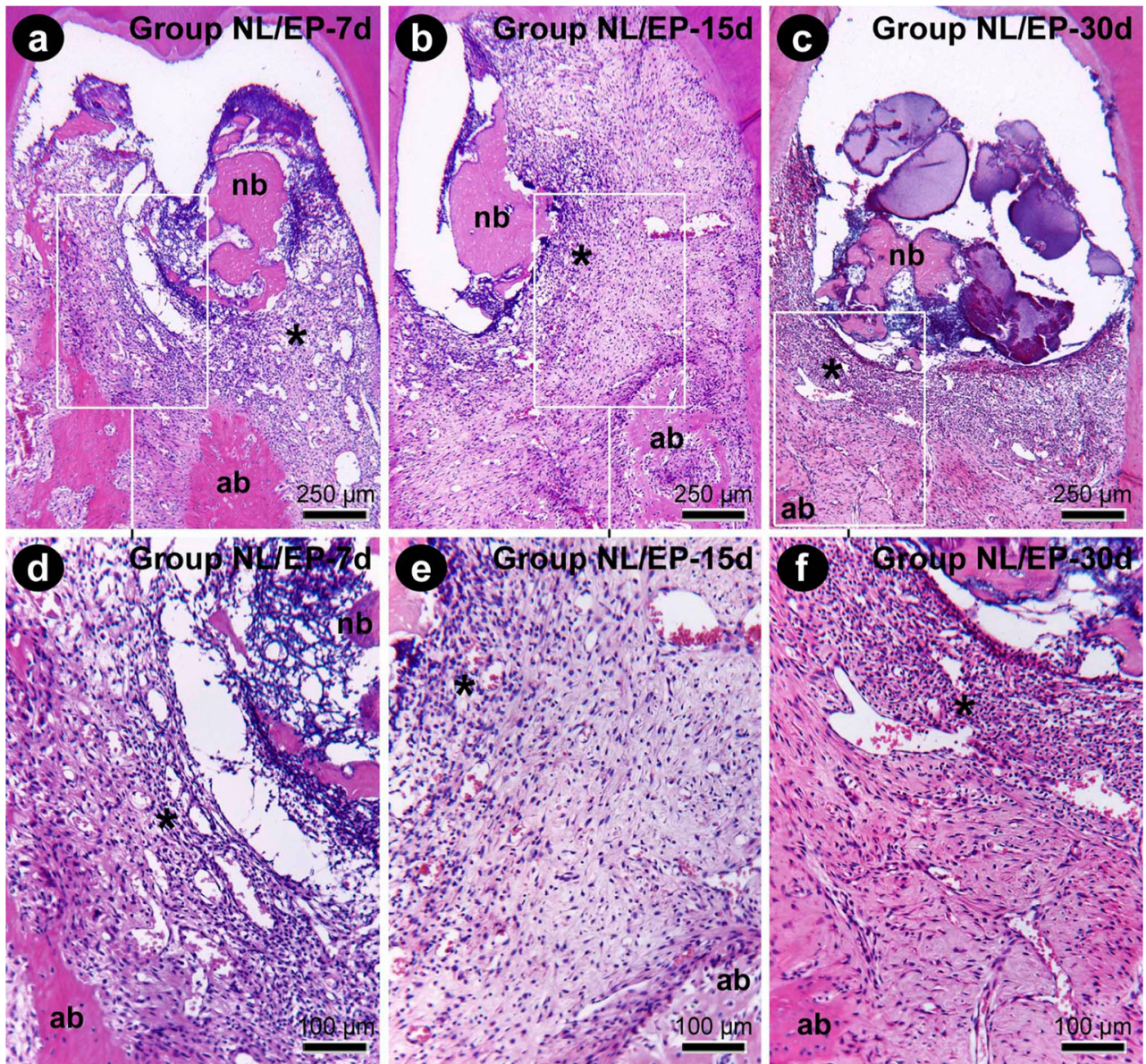

experimental periodontitis than the control groups (NL/C and $\mathrm{HL} / \mathrm{C})$ at all experimental time points $(7,15$, and 30 days) $(p<0.05)$. The intergroup analysis for $\mathrm{EP}(\mathrm{NL} / \mathrm{EP} \times \mathrm{HL} / \mathrm{EP})$ indicated more BL in the HL/EP group than the NL/EP group at 7 days $(p<0.05)$.

\section{Immunohistochemical analysis}

The immunohistochemical method used for the detection of RANKL, TRAP, and OPG yielded high specificity in the detection of these proteins, as evidenced by the total absence of labeling in the negative control. Immunoreactive cells and the extracellular matrix exhibited a brownish color. Figure 6 shows similar amount of osteoclasts TRAP-positive in the $\mathrm{NL} / \mathrm{C}$ and $\mathrm{HL} / \mathrm{C}$ groups and a high number of osteoclasts TRAP-positive in the NL/EP and HL/EP groups. Figure 6 indicates smaller amounts of TRAP-immunoreactive osteoclasts in the NL-C and HL-C groups at 7, 15, and 30 days than the NL-EP and HL-EP. Similar numbers of TRAP-positive cells were verified at 7,15 , and 30 days for the control groups (NL/C and HL/C). The HL/EP group presented greater TRAP-positive cells than the NL/EP at $7(p<0.01), 15$ $(p<0.01)$, and 30 days $(p<0.05)$. The HL/EP group showed the highest number of TRAP-immunoreactivity osteoclasts at 7 days.

There was a high pattern of immunolabeling for RANKL after 7 days of removing the ligature and a low pattern of immunolabeling for OPG after 15 days of removing the ligature in the NL/EP and HL/EP groups (Fig. 7). Immunolabeling for RANKL and OPG was predominantly expressed in cells in the bone and connective tissue in the furcation region. The immunolabeling for RANKL was low (score 1) in the groups without EP and moderate (score 2) in the NL/EP group at all time points. RANKL was high in the HL/EP group (score 3) at 7 and 15 days and moderate at 30 days. There was a statistically significant difference among $\mathrm{NL} / \mathrm{C}, \mathrm{HL} / \mathrm{C}$, and HL/EP for RANKL immunolabeling at 7 days $(p<0.05)$. The immunolabeling for OPG was low (score 1) in all groups, with the exception of $\mathrm{NL} / \mathrm{C}$ at 7 days (score 2; Fig. 7).

\section{Discussion}

Our histological results showed that the periodontal attachment exhibited characteristics of a normal pattern between 
Fig. 5 Histopathological appearance in the furcation region of the mandibular molar focusing on the evolution of experimental periodontitis in rats with a highfat diet (group HL/EP). Histopathological features after 7 (a and d), 15 (b and e), and 30 (c and $\mathbf{f}$ ) days post-ligature placement. Note the severe tissue disorganization and the substantial magnitude of the local inflammatory response.

Abbreviation and symbols: ab, alveolar bone; $\mathrm{nb}$, necrotic bone; asterisks: inflammatory infiltrate. Staining: hematoxylin and eosin (HE). Original magnification: ac, $\times 100 ; \mathbf{d}-\mathbf{f}, \times 250$. Scale bars: a-c, $250 \mu \mathrm{m} ; \mathbf{d}-\mathbf{f}, 100 \mu \mathrm{m}$
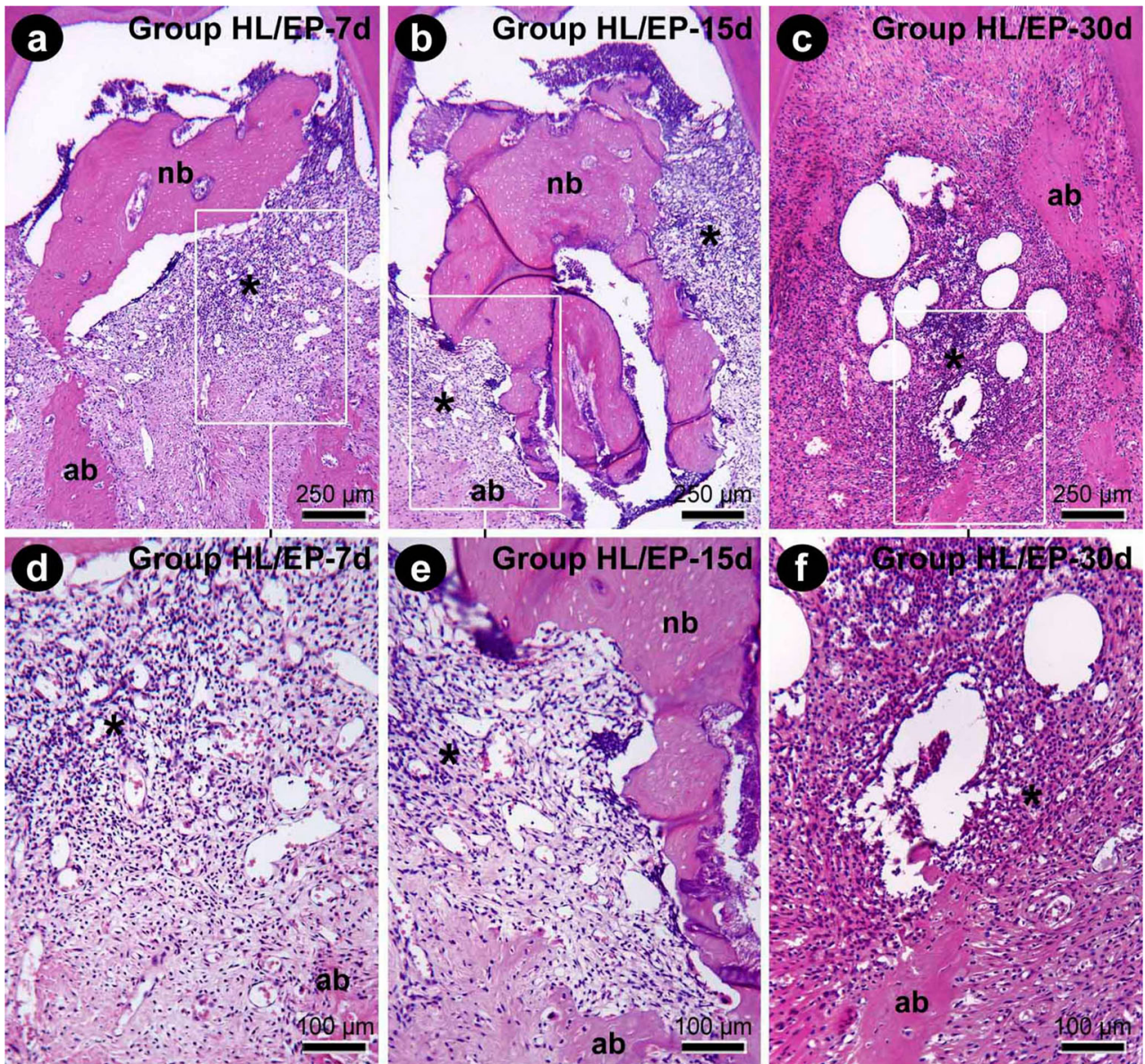

the groups without periodontitis, independent of the presence of induced obesity. The surface of the alveolar bone tissue was full of osteoblasts and had well-delimited bone trabeculae and thick medullary spaces. Therefore, it may be inferred that obesity alone did not cause notable structural changes in

Table 2 Mean and standard deviations $(\mathrm{M} \pm \mathrm{SD})$ of the histometric data for bone loss (BL) in $\mathrm{mm}^{2}$ in the furcation region of the mandibular first molars according to groups and time points

\begin{tabular}{llll}
\hline Groups & \multicolumn{3}{l}{ BL } \\
\cline { 2 - 4 } & 7 days & 15 days & 30 days \\
\hline $\mathrm{NL} / \mathrm{C}$ & $0.12 \pm 0.08^{\text {a A }}$ & $0.07 \pm 0.08^{\text {a A }}$ & $0.25 \pm 0.45^{\text {a A }}$ \\
NL/EP & $1.2 \pm 0.29^{\text {a B }}$ & $0.79 \pm 0.62^{\text {b B }}$ & $1.72 \pm 0.96^{\text {a B }}$ \\
$\mathrm{HL} / \mathrm{C}$ & $0.18 \pm 0.07^{\text {a A }}$ & $0.09 \pm 0.06^{\text {a A }}$ & $0.57 \pm 0.73^{\text {a A }}$ \\
$\mathrm{HL} / \mathrm{EP}$ & $2.28 \pm 0.35^{\text {a C }}$ & $1.4 \pm 0.53^{\text {b B }}$ & $1.21 \pm 1.02^{\text {b B }}$
\end{tabular}

Different lower case letters $(a, b, c)$ indicate statistically significant difference among time points $(7,15$, and 30 days) in the same group (ANOVA and Tukey's test; $p<0.05$ )

Different capital letters (A, B, C) indicate statistically significant difference among groups at the same time point (ANOVA and Tukey's test; $p<0.05)$ periodontal tissues. These results corroborate the morphological findings obtained by Felipetti et al. [22]; however, it must be emphasized that the obesity induction in our study was exogenous, whereas it was endogenous in the previous study. Moreover, it was verified that obesity alone, not associated with EP, was not able to influence greater alveolar bone loss, which confirmed the histological data.

Our results also demonstrated that the local inflammation appears more extensive, with a greater magnitude and longer lasting in the obese animals with EP. In addition, it was noted that the chronicity of the process was delayed in these animals. These same findings were obtained in another study in rats, which indicated that the number of polymorphonuclear leukocytes was higher in the periodontitis combined with obesity group [12]. Other findings showed that in the high-fat dietinduced obesity mice, the periodontal ligament fibers were disrupted, i.e., they lost their orientation with respect to the bone surface, and constriction of the periodontal ligament space was inhibited [23].

There was a statistically significant difference between the groups regarding the bone loss in the furcation region, and the groups with EP showed greater bone loss than their respective controls without ligation. These findings are in agreement 
Fig. 6 Immunolabeling for TRAP in the furcation region of the lower first molars. a Graphic indicates the mean and standard deviations $(\mathrm{M} \pm \mathrm{SD})$ of the TRAP-positive cells in the furcation region of the mandibular first molars according to groups and time points. Different lower case letters (a, b, c) indicate statistically significant difference among time points ( 7 , 15 , and 30 days) in the same group (ANOVA and Tukey's test; $p<0.05)$. Different capital letters (A, B, C) indicate statistically significant difference among groups at the same time point (ANOVA and Tukey's test; $p<0.05$ ). Photomicrographs illustrating the pattern of immunolabeling for TRAP in the groups NL/C (b), HL/C (c), NL/ $\mathrm{EP}$ (d), and HL/EP (e) after 7 days. Note the similar amount of TRAP-positive cells in NL/C (b) and HL/C (c) and the high number of osteoclasts TRAPpositive in NL/EP (d) and specially HL/EP (e).

Abbreviations and symbols: ab, alveolar bone; arrowheads, immunolabeling cells. Counter staining: Harris Hematoxylin. Original magnification $\times 1000$. Scale bars $30 \mu \mathrm{m}$
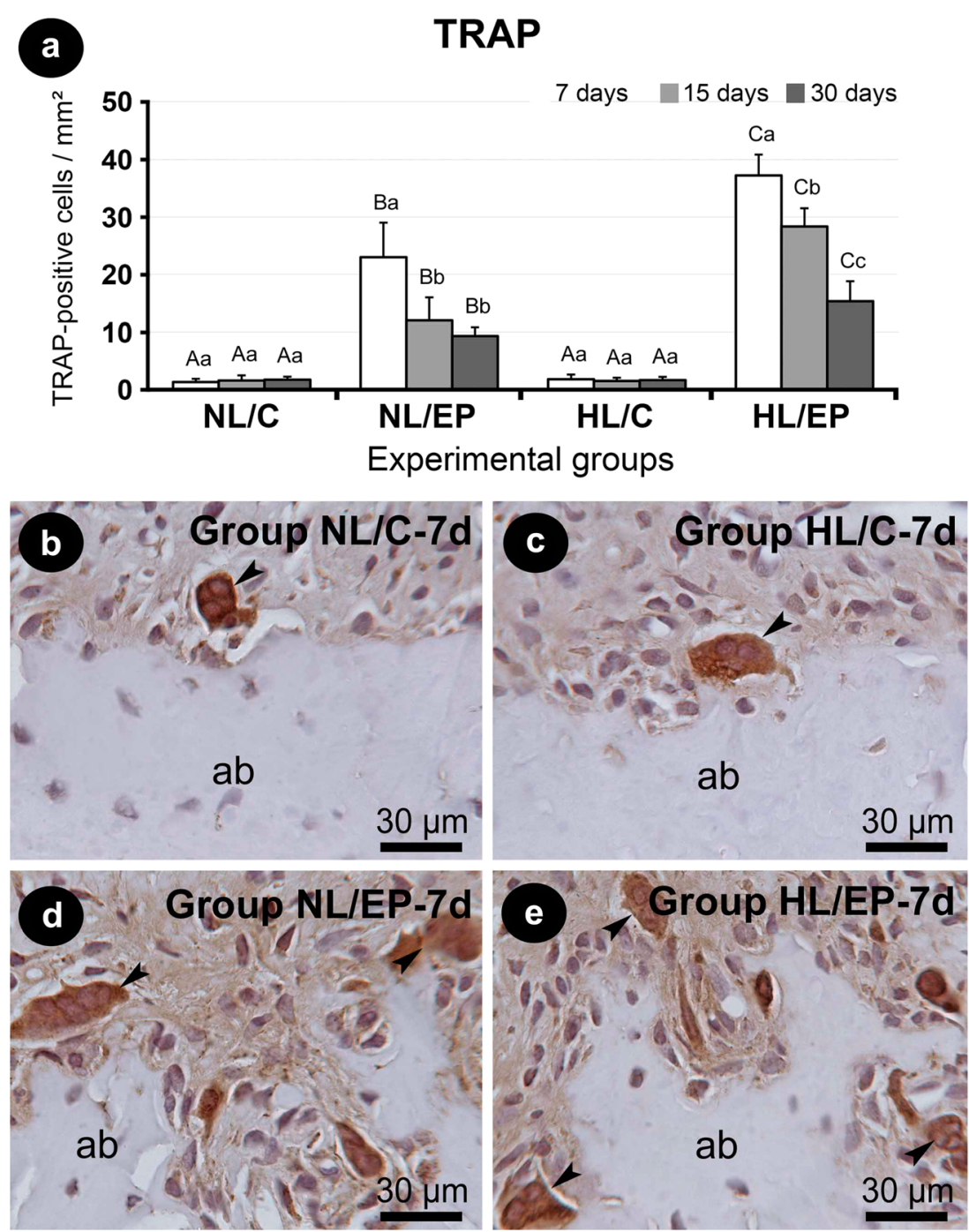

with the results of other experimental studies $[10,12,16,18$, 24]. Moreover, the alveolar bone loss was greater in the rats with obesity associated with EP, which is also in accordance with previous studies, thus suggesting that obesity may increase the severity of periodontal disease [9, 10]. High-fat diet-induced obesity may trigger alveolar bone loss and increase the risk of spontaneous periodontal disease in growing mice [23]. However, other authors did not identify an increase in alveolar bone loss in obese rats with periodontitis compared with non-obese rats [11-13]. These differences in results may be explained by methodological differences because some authors used histometric analyses [12], whereas other authors used morphometric measurements [11]. Furthermore, a recent meta-analysis indicated that whether patients who exhibit chronic periodontitis with obesity have elevated proinflammatory gingival crevicular fluid biomarker levels compared with non-obese subjects remains debatable [25].

The greatest bone loss observed in the rats with obesity and EP occurred at the 7 day time point $(p<0.05)$, and it decreased by the 15 and 30 day time points. One hypothesis that may explain our findings is related to the study by Amar et al. [9]. These authors demonstrated that rats with obesity and induced periodontitis showed a delay in the response to TNF- $\alpha$ serum in response to Porphyromonas gingivalis (P.g.), with the highest peak on the 7th day and a significant cancelation over the following days. This finding may indicate an attenuated systemic immune response to P.g. in the group with induced obesity, which supports the hypothesis that obesity may alter the innate immune response to P.g.; however, it undermines its role in the elimination or aggravation of the periodontal bone loss. These results are understandable because there is compensation in the host organism's defense mechanism during longer periods of EP progression compared with the initial periods of EP [26]. Furthermore, there are obesity-related molecules, such as adipokines, which are bioactive molecules secreted by adipose tissue that regulate the inflammatory and healing processes. Thus, since visfatin and leptin levels are increased and adiponectin levels are reduced in obesity, these 
Fig. 7 Immunolabeling for RANKL and OPG in the furcation region of the lower first molars. Graphics indicate the median and first and third quartiles of the RANKL (a) and OPG scores (f) in the furcation region of the mandibular first molars according to groups and time points. Photomicrographs illustrating the pattern of immunolabeling for RANKL (be) and OPG $(\mathbf{g}-\mathbf{j})$ in the groups $\mathrm{NL} / \mathrm{C}$ (b and $\mathbf{g}$ ), HL/C (c and $\mathbf{h}$ ), NL/EP (d and i), and HL/EP (e and j). A high pattern of immunolabeling for RANKL was observed after 7 days of removing the ligature, and a low pattern of immunolabeling was observed for OPG after 15 days of removing the ligature in the NL/EP and HL/ EP groups. Abbreviations and symbols: ab, alveolar bone; arrowheads, immunolabeling cells. Counter staining: Harris Hematoxylin. Original magnification: $\times 1000$. Scale bars $30 \mu \mathrm{m}$. *Difference compared to the NL/C and HL/C at 7 days $(p<0.05$; Kruskal-Wallis and Dunn test) a

7 days $\square 15$ days $\square 30$ days
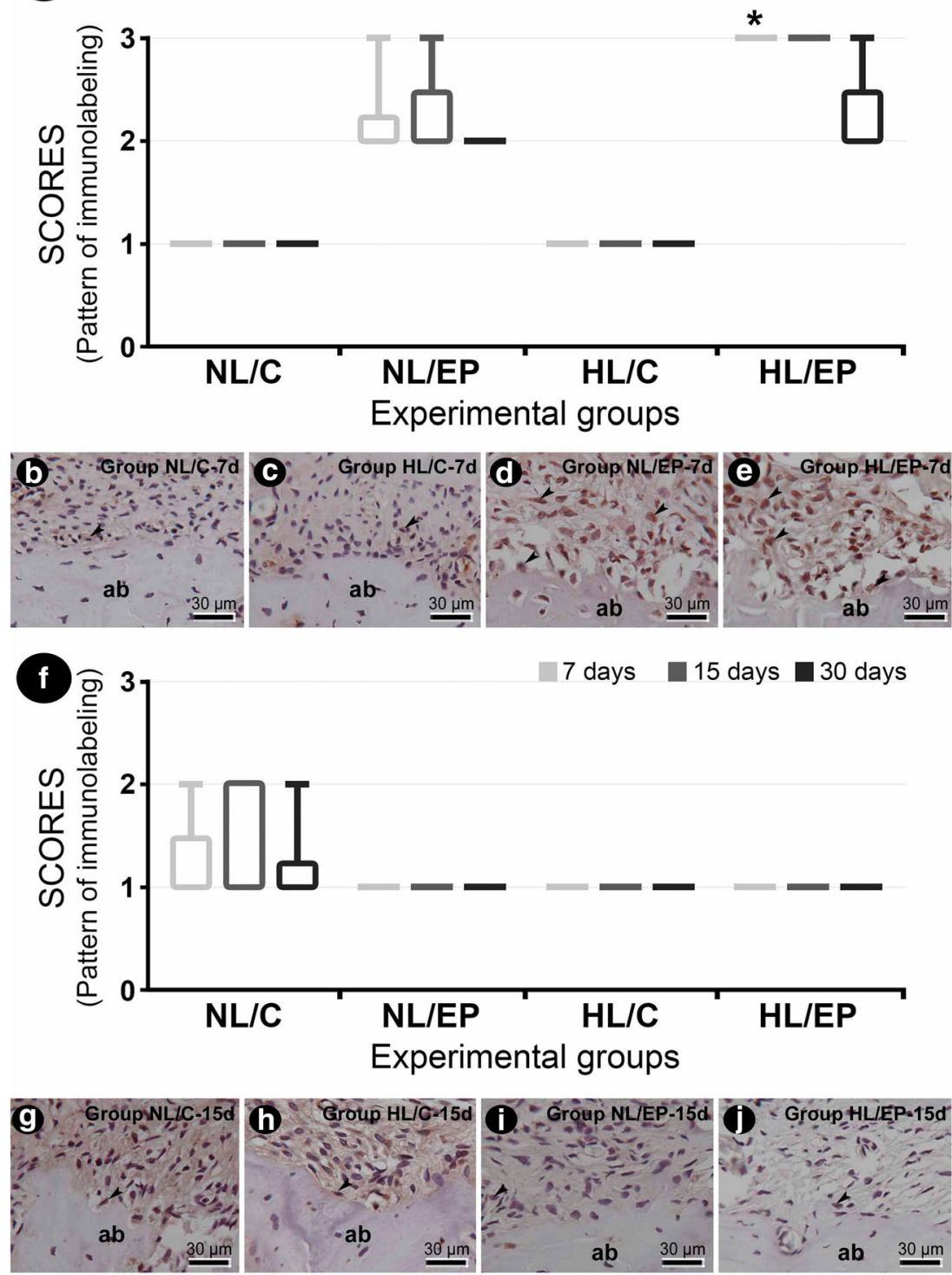

7 days

15 days

30 days
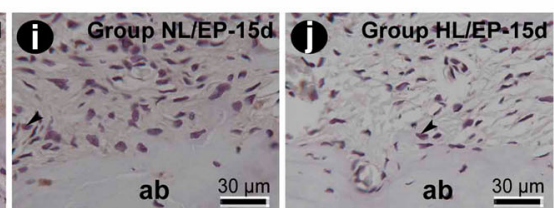

adipokines may be a pathomechanistic link by which obesity and obesity-related diseases enhance the risk for periodontitis and compromised periodontal healing [27].

Our data showed higher immunolabeling of TRAP and RANKL in the initial periods of periodontitis in both groups ( $\mathrm{HL}$ and $\mathrm{NL}$ ), and the periodontal inflammatory response was more pronounced in obese animals (HL). The animals in the HL/EP group presented greater TRAP-positive cells than the animals in the NL/EP group. RANKL was high in the HL/EP group (score 3) at 7 and 15 days and moderate at 30 days. There was greater RANKL immunolabeling in HL/EP than in NL/EP at 7 days. These results suggest that obesity is capable of effectively influencing the recruitment and activity of bone tissue and connective tissue cells in the periodontium, as well as increasing the severity of periodontal disease in the experimentally induced sites. This finding is in line with previous studies [28] that have reported an exacerbated systemic inflammation in obese individuals with an increased production of proinflammatory cytokines, such as interleukin 1 (IL-1), interleukin 6, and tumor necrosis factor alpha, by adipocytes, which are able to promote osteoclast activity by regulation of the RANK/RANKL/OPG system. Similarly, adipocytes may directly regulate hematopoietic progenitor cells of osteoclasts [29], which may explain why we observed the increase in the recruitment of osteoclasts and greater bone loss in the HL/EP group. A recent study also showed that the effect of central adiposity on future periodontitis progression is conditional on proinflammatory IL-1 genetic variations [30].

Several studies have combined this model of EP with models of obesity induction by a hypercaloric diet to achieve satisfactory weight gain in rats to assess the relationship between periodontal disease and obesity and identify potential 
mechanisms involved [9-13]. Our findings corroborate these studies and showed that the hypercaloric diet was able to induce weight gain in the animals tested compared with animals fed standard animal food. This approach has been a good model for the induction of exogenous obesity, whereas endogenous obesity may be induced by other experimental models, such as the subcutaneous injection of monosodium glutamate; however, this method may generate conditions that are not ideal for investigations of the development and progression of periodontal disease [22]. Other authors have also assessed EP in models with obese Zucker rats that bear a recessive autosomal mutation for the leptin receptor gene, which causes chronic hyperphagia and morbid obesity [12].

The timing of obesity induction should also be taken into account as different stages of obesity may lead to different severities of systemic inflammation [31-33]. In this study, we employed a time period of 16 weeks to induce obesity, whereas previous studies have shown that less than 8 weeks of feeding with a hypercaloric diet resulted in mild systemic inflammation [31] or 16 or more weeks of induction resulted in moderate to morbid obesity, with a moderate or severe inflammatory response $[32,33]$. This variation in the induction time of obesity may explain the inconsistencies between studies. For example, in a study by Simch et al. [11], the authors suggested that the establishment and progression of ligature-induced alveolar bone loss in rats was not influenced by body weight; however, the animals showed only an overweight condition because obesity was not reached.

Our study is one of the first investigations to show the effects of obesity on periodontal disease with bone markers, such as RANKL, OPG, and TRAP. We also demonstrate that obesity affects the regulation of bone metabolism when associated with periodontal disease. Future studies should be performed to assess the effect of periodontitis on the organs and tissues involved.

Within the limits of this study, it may be concluded that the joint effect of obesity induced by a high-fat diet and periodontitis influenced the alveolar bone metabolism with greater bone loss, prolonged exacerbation of periodontal inflammatory response and a positive modulation of ostoclastogenesis and osteoclast activity via RANKL upregulation.

Acknowledgements We thank the vivarium technician Darcy Thomaz de Aquino (in memoriam) and Professor Patrícia Rodella for the laboratory analyses at UNIFEB.

Funding This study was financially supported by the São Paulo State Research Foundation-FAPESP, São Paulo, SP, Brazil (process no. 2011/08053-0).

\section{Compliance with ethical standards}

Conflict of interest The authors have no conflicts of interest.
Ethical approval All applicable international, national, and/or institutional guidelines for the care and use of animals were followed.

Informed consent For this type of study, formal consent is not required.

\section{References}

1. Williams RC (1990) Periodontal disease. N Engl J Med 322:373382

2. Graves DT, Li J, Cochran DL (2011) Inflammation and uncoupling as mechanisms of periodontal bone loss. J Dent Res 90:143-153

3. Zuza EP, Barroso EM, Carrareto ALV, Pires JR, Carlos IZ, Theodoro LH, Toledo BE (2011) The role of obesity as a modifying factor in patients undergoing non-surgical periodontal therapy. J Periodontol 82:676-682

4. Ritchie CS (2007) Obesity and periodontal disease. Periodontol 2000(44):154-163

5. Boesing F, Patiño JSR, da Silva VRG, Moreira EAM (2009) The interface between obesity and periodontitis with emphasis on oxidative stress and inflammatory response. Obes Rev 10:290-297

6. Pischon N, Heng N, Bernimoulin J-P, Kleber B-M, Willich SN, Pischon T (2007) Obesity, inflammation, and periodontal disease. J Dent Res 86:400-409

7. Chaffee BW, Weston SJ (2010) Association between chronic periodontal disease and obesity: a systematic review and meta-analysis. J Periodontol 81:1708-1724

8. Suvan J, D'Aiuto F, Moles DR, Petrie A, Donos N (2011) Association between overweight/obesity and periodontitis in adults. A systematic review. Obes Rev 12:381-404

9. Amar S, Zhou Q, Shaik-Dasthagirisaheb Y, Leeman S (2007) Dietinduced obesity in mice causes changes in imune responses and bone loss manifested by bacterial challenge. Proc Natl Acad Sci U S A 104:20466-20471

10. Nascimento CM, Cassol T, Silva FS, Bonfleur ML, Nassar CA, Nassar PO (2013) Radiographic evaluation of the effect of obesity on alveolar bone loss in rats with ligature-induced periodontal disease. Diabetes Metab Syndr Obes 6:365-370

11. Simch RP, Gaio EJ, Rösing CK (2008) Effect of body weight in the pathogenesis of ligature-induced periodontal disease in Wistar rats. Acta Odontol Scand 66:130-134

12. Endo Y, Tomofuji T, Ekuni D, Irie K, Azuma T, Tamaki N, Yamamoto T, Morita M (2010) Experimental periodontitis induces gene expression of proinflammatory cytokines in liver and white adipose tissues in obesity. J Periodontol 81:520-526

13. Verzeletti GN, Gaio EJ, Linhares DS, Rösing CK (2012) Effect of obesity on alveolar bone loss in experimental periodontitis in Wistar rats. J Appl Oral Sci 20:218-221

14. Cabeço LC, Akiba M, Calsa MS, Sartori DRS, Vicentini-Paulino MLM, Pinheiro DF (2010) High-fat diet with soy flour as protein source: use in selection of mice prone and obesity resistant (in Portuguese). Rev Nutr 23:417-424

15. Dong H, Altomonte J, Morral N, Meseck M, Thung SN, Woo SL (2002) Basal insulin gene expression significantly improves conventional insulin therapy in type 1 diabetic rats. Diabetes 51:130 138

16. Almeida JM, Theodoro LH, Bosco AF, Nagata MJH, Bonfante S, Garcia VG (2008) Treatment of experimental periodontal disease by photodinamic therapy in rats with diabetes. J Periodontol 79: 2156-2165

17. Garcia VG, Longo M, Gualberto Júnior EC, Bosco AF, Nagata MJ, Ervolino E, Theodoro LH (2014) Effect of the concentration of phenothiazine photosensitizers in antimicrobial photodynamic 
therapy on bone loss and the immune inflammatory response of induced periodontitis in rats. J Periodontal Res 49:584-594

18. Gomes DA, Pires JR, Zuza EP, Muscara MN, Herrera BS, Spolidorio LC, Toledo BE, Spolidorio DM (2009) Myeloperoxidase as inflammatory marker of periodontal disease: experimental study in rats. Immunol Investig 38:117-122

19. Gomes DA, Spolidorio DM, Pepato MT, Zuza EP, de Toledo BE, Gonçalves A, Spolidorio LC, Pires JR (2009) Effect of induced diabetes mellitus on alveolar bone loss after 30 days of ligatureinduced periodontal disease. J Int Acad Periodontol 11:188-192

20. Lisboa MR, Gondim DV, Ervolino E et al (2015) Effects of electroacupuncture on experimental periodontitis in rats. $\mathrm{J}$ Periodontol 86:801-811

21. Kim YD, Kim SS, Hwang DS et al (2007) Effect of low-level laser treatment after installation of dental titanium implantimmunohistochemical study of RANKL, RANK, OPG: an experimental study in rats. Lasers Surg Med 39:441-450

22. Felipetti FA, Silva EO, Rangel ALCA et al (2014) Evaluation of alveolar bone mandibular of rats obese induced at monosodium glutamate undergoing modified duodenal-jejunal bypass surgery. $\mathrm{J}$ Endocrinol Metab 14:93-100

23. Fujita Y, Maki K (2016) High-fat diet-induced obesity triggers alveolar bone loss and spontaneous periodontal disease in growing mice. BMC Obes 3:1

24. Perlstein MI, Bissada NF (1977) Influence of obesity and hypertension on the severity of periodontitis in rats. Oral Surg Oral Med Oral Pathol 43:707-719

25. Akram Z, Abduljabbar T, Abu Hassan MI, Javed F, Vohra F (2016) Cytokine profile in chronic periodontitis patients with and without obesity: a systematic review and meta-analysis. Dis Markers 2016: 4801418

26. Almeida JM, Theodoro LH, Bosco AF, Nagata MJ, Oshiiwa M, Garcia VG (2007) Influence of photodynamic therapy on the development of ligature-induced periodontitis in rats. J Periodontol 78:566-575

27. Deschner J, Eick S, Damanaki A, Nokhbehsaim M (2014) The role of adipokines in periodontal infection and healing. Mol Oral Microbiol 29:258-269

28. Khosla S (2001) Minireview: the OPG/ RANKL/RANK system. Endocrinol 142:5050-5055

29. Naveiras O, Nardi V, Wenzel PL, Hauschka PV, Fahey F, Daley GQ (2009) Bone-marrow adipocytes as negative regulators of the haemotopoietic microenvironment. Nature 460:259-263

30. Wilkins LM, Kaye EK, Wang HY, Rogus J, Doucette-Stamm L, Komman K, Garcia RI (2017) Influence of obesity on periodontitis progression is conditional on interleukin-1 inflammatory genetic variation. J Periodontol 88:59-68

31. Stanton MC, Chen SC, Jackson JV et al (2011) Inflammatory signals shift from adipose to liver during high fat feeding and influence the development of steatohepatitis in mice. J Inflamm 8:8

32. $\mathrm{Xu} \mathrm{H}$, Barnes GT, Yang Q et al (2003) Chronic inflammation in fat plays a crucial role in the development of obesity-related insulin resistance. J Clin Invest 112:1821-1830

33. Buckman LB, Hasty AH, Flaherty DK et al (2014) Obesity induced by a high-fat diet is associated with increased immune cell entry into the central nervous system. Brain Behav Immun 35:33-42 